\title{
Information impact on quality of multimodal travel choices: conceptualizations and empirical analyses
}

\author{
Caspar G. Chorus · Theo A. Arentze · Harry J. P. Timmermans
}

Published online: 17 May 2007

(C) Springer Science+Business Media B.V. 2007

\begin{abstract}
This paper investigates the impact of a variety of travel information types on the quality of travel choices. Choice quality is measured by comparing observed choices made under conditions of incomplete knowledge with predicted choice probabilities under complete knowledge. Furthermore, the potential impact of travel information is considered along multiple attribute-dimensions of alternatives, rather than in terms of travel time reductions only. Data is obtained from a choice experiment in a multimodal travel simulator in combination with a web-based mode-choice experiment. A Structural Equation Model is estimated to test a series of hypothesized direct and indirect relations between a traveler's knowledge levels, information acquisition behavior and the resulting travelchoice quality. The estimation results support the hypothesized relations, which provides evidence of validity and applicability of the developed measure of travel-choice quality. Furthermore, found relations in general provide some careful support for the often expected impact of information on the quality of travel choices. The effects are largest for information services that generate previously unknown alternatives, and lowest for services that provide warnings in case of high travel times only.
\end{abstract}

Keywords Travel information - Travel choice quality $\cdot$ Structural equation model

\section{Introduction}

The interest in Advanced Traveler Information Services (ATIS) has grown rapidly over the last years, already culminating in a large body of academic literature on this topic (see for some notable contributions Ben-Akiva et al. 1991, Polak and Jones 1993, Emmerink et al.

C. G. Chorus $(\bowtie) \cdot$ T. A. Arentze · H. J. P. Timmermans

Urban Planning Group, Eindhoven University of Technology, P.O. Box 513, 5600 MB, Eindhoven, The Netherlands

e-mail: c.g.chorus@tue.nl 
1995, 1996, Hato et al. 1999, Denant-Boèmont and Petiot 2003 or for a review Chorus et al. 2006a). Furthermore, a stream of policy initiatives has been built on the deployment of ATIS in transport networks (e.g. Commission of the European Communities 2001; Ministry of Transport, Public Works and Water Management 2002; Department for Transport 2004). A main underlying justification for this large and growing interest, and for the huge investments needed for the development and deployment of such services, is the following line of argumentation: multimodal urban transport networks become more and more complex and unreliable, so that it becomes practically impossible for travelers to attain complete knowledge of all relevant factors influencing travel choice. This lack of knowledge predominantly exists along two dimensions (Bonsall 2004; Chorus et al. 2006b): firstly, travelers may not know all available and feasible travel alternatives (in terms of mode-route combinations) that may bring them to their destination (e.g. Ramming 2002; Hoogendoorn-Lanser and Van Nes 2004). Secondly, of the alternatives they do know, they may not know their precise attributes in terms of travel times, costs and other relevant factors (e.g. Ouwersloot et al. 1997; Bonsall 2001; Rietveld et al. 2001; Bates et al. 2001; Avineri and Prashker 2003). These conditions of incomplete knowledge are assumed to lead to decreasing quality of travel choices. It is hoped for, and by many expected, that travel information provision may assist travelers in making better choices in these increasingly uncertain and complex multimodal travel networks by increasing travelers' knowledge levels (e.g. Khattak et al. 1993; Walker and Ben-Akiva 1996; Adler and Blue 1998; Golledge, 2002; Abdel-Aty and Abdalla 2004; Jou et al. 2005).

One would expect that a substantial body of knowledge has been acquired over the years to test whether these expectations are correct. A first body of research, based on empirical data obtained from surveys or choice experiments, has focused on travelers' subjective perceptions of the potential of information to improve their choices. These research efforts either studied travelers' willingness to pay for information (e.g. Polydoropoulou et al., 1997; Khattak et al. 2003; Denant-Boèmont and Petiot 2003; Molin and Chorus 2004) or their ex-post judgments of how available information helped them make better choices (Chatterjee and McDonald, 2004; Benjamin 2006). These approaches, notwithstanding the valuable insights they present, lack an objective measurement ${ }^{1}$ of information impact on choice quality. This disadvantage is addressed in a second body of research, that studies the potential of information to improve travelers' choices by deriving theoretical models and illustrating these with numerical examples based on synthetic data (e.g. Mahmassani and Jayakrishnan, 1991; Arnott et al. 1991; Al-Deek et al. 1998; Levinson 2003; Gentile et al. 2005; Szeto and Lo 2005). This latter approach allows the objective measurement of information impact on choice quality (e.g. in terms of mean travel time reductions), but suffers from an inherent lack of external validity as no real life behavior is observed. Exceptions are found in Adler (2001) and Bogers et al. (2005), where the impact of information on travel times is studied empirically. All these research efforts have focused on either travel by car (predominantly) or by transit, and were predominantly concerned with travel time information only, and its effect on travelers' realized travel times. This limited scope fails to acknowledge that travelers generally make their choices in a

\footnotetext{
1 With the term objective measurement we refer in this paper to a measurement that is based on a comparison by the analyst of choices made with, and those made without, travel information. Such measurements contrast with subjective measurements, where choice makers themselves state the expected or experienced impact of information on the quality of their choices.
} 
multimodal environment where a variety of travel information types might be available to them, and that they generally base their choices on many more attributes than travel times only.

This paper presents a study that aims at (1) combining the advantages of the different streams of research presented above, and at (2) providing a broader scope of research, one that better fits the full dimensionality of traveler behavior. This is done by empirically investigating the impact of a variety of travel information types, including the assessment of known alternatives and the generation of unknown alternatives, on the quality of observed multimodal travel choices. Furthermore, an objective measurement of choice quality is applied that is based on the following general notion: a traveler's choice for an alternative (e.g. a mode-route combination), made under incomplete knowledge, is of a high quality if the traveler, given complete knowledge, would choose the same alternative. Finally, the potential impact of travel information is considered along multiple attributedimensions of alternatives, such as travel times and travel costs of car and transit alternatives, as well as seat probabilities in transit. Data needed for empirical analysis is obtained from a choice experiment in a multimodal travel simulator with information provision, where 261 participants together made 4536 travel choices under conditions of incomplete knowledge, in combination with a web-based mode-choice experiment where the same participants made choices under conditions of complete knowledge.

The remainder of the paper is structured as follows: in "Hypotheses on travel choice quality and the role of travel information" we briefly illustrate the proposed general notion of travel choice quality with an example. Subsequently, hypotheses are formulated that are to be tested in this study; they relate to the determinants of travel choice quality in general and the role of information acquisition in specific. "Data-requirements and -collection" discusses data requirements that follow from the hypotheses to be tested and the general notion of choice quality proposed here. Subsequently, the data-collection effort is presented. "A measure of travel choice quality" derives from the proposed general notion an operational measure of travel choice-quality that is compatible with the obtained data. "Empirical analysis of the impact of travel information on travel choice quality" presents the empirical analysis of the impact of travel information on travel choice quality by estimating a structural equation model that is based on the obtained choice data and the derived choice quality measure. Estimation results are discussed in depth. Finally, "Conclusions and discussion"' presents conclusions and directions for further research.

\section{Hypotheses on travel choice quality and the role of travel information}

Before formulating hypotheses concerning the determinants of travel choice quality, let us briefly discuss the general notion of travel choice quality that is presented in the introduction. We conceptualize travel choice quality as the extent to which a traveler's choice is the same under conditions of incomplete knowledge and complete knowledge. The following brief example may illustrate this conceptualization: take a traveler that faces a choice under conditions of incomplete knowledge. That is, she knows of only some of the available alternatives (e.g. routes for a particular mode) and for the alternatives she knows of, she does not know their exact attributes (e.g. travel times and costs). Based on this incomplete knowledge, she chooses a mode-route combination from the set of known alternatives. Given our conceptualization, this choice has a high quality when the traveler, would she have known all available alternatives as well as the exact value of their attri- 
butes, would choose the same route. Otherwise, her lack of knowledge has resulted in a choice of relatively low quality. Note how the adopted notion accounts for a travelers' preferences: assume that the traveler of our example has a very low value of time, and is uncertain about alternatives' travel times but not about their costs. In that case travel time uncertainty will not lead to large reductions in the quality of her choices, as conceptualized here, since these choices are not strongly influenced by travel times. Therefore, the probability that she would have chosen a different mode-route combination under the hypothetical condition of travel time certainty is relatively low. Due to its generality, this notion is well suited for testing hypotheses concerning the impact of a wide variety of factors on travel choice quality, along multiple attribute dimensions. We will specifically address in this study the following hypotheses:

1. The more alternatives a traveler knows of, the higher will be her travel choice quality Not only is this an intuitive relation, it in fact is implied by the adopted general notion of choice quality, as it is presented in the introduction: the more alternatives a traveler knows of, the lower will be the probability (ceteris paribus) that one of the remaining unknown alternatives is in fact the best one available, and would be chosen under conditions of complete knowledge. A test of this hypothesis therefore foremost forms a test of the validity of the general notion of choice quality proposed here.

2. The less uncertainty is attached to the attributes of known alternatives, the higher will be a traveler's choice quality (given that there is no structural bias in perceptions)Similar to hypothesis 1 , this one is also implied by the adopted notion of choice quality: the more certain the choice-maker is about the actual value of the attributes of known alternatives, the less likely she is to choose an alternative from this set that she would not have chosen under conditions of complete knowledge. A test of this hypothesis therefore also forms a test of the validity of the general notion of choice quality proposed here.

3. The more travel information is acquired, the higher will be the travel-choice quality This expected relation is the central hypothesis of the study presented here. Although this notion may appear evident to some, there is in fact little empirical evidence to support this expectation, as was argued in the introduction. It may be expected that different types of information may have different impacts on the quality of travel choices.

4. The more complete a traveler's knowledge, the less information will be acquiredMuch empirical work has been done on this topic (e.g. Hato et al. 1999; Yim and Khattak 2002; Denant-Boèmont and Petiot 2003; Targa et al. 2003; Petrella and Lappin 2004; Peirce and Lappin 2004; Van der Horst and Ettema 2005; Chorus et al., forthcoming). Generally, these studies point out that lack of knowledge does drive information acquisition, but that subtle effects may exist in the opposite direction. An example of such a subtle mechanism is that the more alternatives a traveler knows of, the more inclined she might become to assess the attributes of these known alternatives.

5. For business trips, travelers acquire more information and, controlled for the acquired information, make travel choices of relatively high qualityThe first part of this hypothesis is well established in empirical literature on travel information acquisition for arrival time sensitive trips (e.g. Emmerink et al., 1996; Polydoropulou and BenAkiva, 1998; Hato et al. 1999; Srinivisan et al. 1999), and makes sense intuitively. The second part is based on the notion that when the stakes are high (in this case: the penalty of late arrival is high), individuals may put more effort in their decision 
making process which in turn may help them make better choices (e.g. Payne et al. 1993).

6. The more unreliable the information, the less it is acquired and when it is acquired, the less will be its positive impact on travel choice quality. The importance of (perceived) information reliability as a determinant of information use is well grounded in empirical literature (Polydoropulou and Ben-Akiva, 1998; Hato et al., 1999; Fayish and Jovanis 2004). The second part of the hypothesis is based on the notion, found in theoretical studies of travel information effect (e.g. Arenzte and Timmermans 2005; Chorus et al., 2006b), that due to unreliability of the information, travelers may only partly reduce the uncertainty of their initial perceptions.

\section{Data-requirements and -collection}

Derivation of data-requirements

Given the adopted conceptualization of travel choice quality and the formulated hypotheses, a range of data requirements can be derived: firstly, we need to observe travel choices made under conditions of various levels of incomplete knowledge, in terms of both small numbers of known alternatives and large levels of uncertainty with respect to their attributes. In this context of incomplete knowledge, a wide range of travel information should be available to the traveler. Furthermore, we as an analyst need to be able to observe the set of available alternatives and the true values of their attributes. The needed level of experimental control (i.e. the need to be able to control travelers' knowledge levels) in combination with the need to provide a variety of travel information options led us to the deployment of a multimodal travel simulator with information provision (Chorus et al., 2006c), see "Travel simulator experiment (observation of choices under incomplete knowledge)', Finally, we need to infer what choices travelers would have made if they would have had complete knowledge in the same choice situations. Given the hypothetical nature of the condition, the latter choices cannot be directly observed. Therefore, we predict them based on estimated preferences of travelers for alternatives and their attributes and assuming complete knowledge. A web-based stated mode-choice experiment under conditions of complete knowledge is held among the same group of respondents that participated in the simulator experiment, during the same session, to derive estimates for their preferences ("Stated mode-choice experiment (observation of choices under complete knowledge)'”).

Response group characteristics and outline of the experiment

Participants were recruited through placement of advertisements in a campus newspaper and a free newspaper. Also an email was sent out to \pm 500 students. Criterion for participation was that participants had some experience with traveling by both car and train. A 20 euro reward was offered for participation. In total, 261 individuals were recruited this way. Table 1 presents some response group characteristics and shows a rather heterogeneous group in terms of socio-economic characteristics. However, it should be noted that the participants were not average travelers due to the above mentioned selection criterion. This should be taken into account when interpreting the results of the analysis presented 
Table 1 Response group characteristics $(N=261)$

\begin{tabular}{|c|c|c|}
\hline Variable & Frequency $(\%)$ & Cumulative (\%) \\
\hline \multicolumn{3}{|l|}{ Gender } \\
\hline Female & 49 & 49 \\
\hline Male & 51 & 100 \\
\hline \multicolumn{3}{|l|}{ Age } \\
\hline$<25$ & 45 & 45 \\
\hline$<40$ & 27 & 72 \\
\hline$<65$ & 27 & 99 \\
\hline$>65$ & 1 & 100 \\
\hline \multicolumn{3}{|l|}{ Completed education } \\
\hline Lower education & 2 & 2 \\
\hline Secondary school & 67 & 69 \\
\hline Higher education & 31 & 100 \\
\hline \multicolumn{3}{|c|}{ Main out-of-home activity } \\
\hline Paid work & 41 & 41 \\
\hline Education & 45 & 86 \\
\hline Other & 14 & 100 \\
\hline \multicolumn{3}{|l|}{ Car availability } \\
\hline Always & 39 & 39 \\
\hline Usually & 18 & 57 \\
\hline Sometimes & 26 & 83 \\
\hline Less than sometimes & 17 & 100 \\
\hline \multicolumn{3}{|c|}{ Public transport (PT) season ticket } \\
\hline Some form of & 62 & 62 \\
\hline None & 38 & 100 \\
\hline
\end{tabular}

further below, although it is not directly clear what potential biases our sample-selection might induce. The sessions each followed the same program. After a brief introduction, an extensive web survey was filled out concerning among other things the participant's actual travel behavior and his or her acquisition of travel information through different media. Following this web survey, a stated choice experiment was performed (see "Stated modechoice experiment (observation of choices under complete knowledge)"'), after which the participants were given a rather extensive introduction in the travel simulator's workings (see "Travel simulator experiment (observation of choices under incomplete knowledge)'”).

Following this, the participants were given a rather extensive introduction in the simulator. Participants were asked very explicitly to not regard the experiment as some form of a game (e.g. by trying to travel as fast as possible, or spending as much or as little money as possible), but rather to try to identify with the travel situations presented and make choices that they would make, would they be confronted with such a situation in real life. It is known that in simulated travel situations like the ones presented in this experiment, the issue of motivation is a difficult one. See Carson et al. (2000) and Bonsall (2002) for overviews of possible incentive-caveats. In order to increase the motivation of participants to put effort in identifying themselves with the simulated travel environment, the following approach was chosen: participants were told during the introduction that they could win a 
7.5 euro bonus, to be awarded to about half of the respondents, based on the success of their identification effort. It was mentioned that the correspondence of their choicebehavior as observed in the simulator experiment with the choice-behavior observed in the stated mode choice experiment and the answers to web-survey questions concerning revealed behavior would be used to measure the degree of identification. However, it was made clear to the participants that they would probably be most likely to obtain the bonus by simply making a real effort to identify with each of the travel situations presented. After this introduction, participants all made four test-rides, two with and two without the availability of travel information. Subsequently, a number of trips (maximum 25) were made in the travel simulator environment and recorded, all in the presence of information services.

Travel simulator experiment (observation of choices under incomplete knowledge)

Figure 1 shows a screen plot of an arbitrary travel situation a participant may be confronted with. The workings of the simulator will be discussed by going through this figure. The screen consists of four parts: lower left presents the trip context, upper-left shows the transport network, upper right presents the information service and lower right shows a visual aid.

\section{Trip purpose}

The trip purpose consists of a story line describing trip purpose and possibly preferred arrival times, generated at random for each trip from a set of predefined options. These story lines were presented at the beginning of each new trip through pop-up windows, after

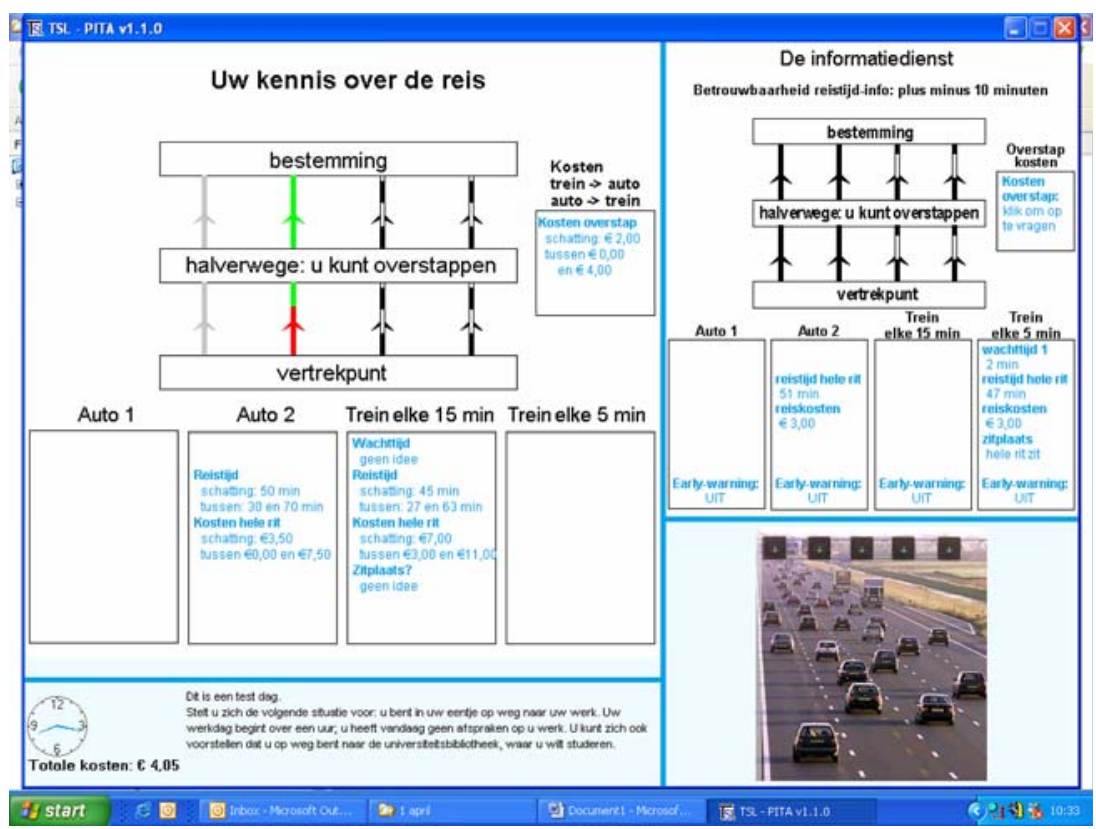

Fig. 1 Screen plot of the simulator (in Dutch) 
which they were located at the lower left of the screen during the completion of the trip. Trip purpose could be either business, commute, social or leisure. Next to the story line, the trip context displays a clock, presenting accelerated time. It ticks away one minute waiting time or in-vehicle time per actual second, after a travel alternative is executed. Finally, beneath the clock, a money counter registers the amount of money spent so far on a given trip, including both travel costs and costs of information acquisition.

\section{The transport network}

A purely fictive O-D pair was created, connected by four paths displayed as arrows and an interchange facility halfway; as our current analyses do not deal with in-trip choice adaptation, we will not discuss here the interchange possibilities. The two left arrows symbolize two car-options, i.e. highway routes. The two routes are equivalent except that they may differ in terms of travel times and costs. Next to these two car-options, two intercity train options exist which are also equivalent, except that they may also differ in travel time and cost, as well as seat availability. Furthermore, the left one of the two trains departs once every $15 \mathrm{~min}$, the right one once every $5 \mathrm{~min}$, thus inducing a lower expected and maximum waiting time. The number of a priori alternatives 'known' to the traveler varies per trip. 'Unknown' alternatives are marked grey instead of black, and the traveler initially has no knowledge concerning their characteristics. These alternatives are inactive, and cannot be executed by the traveler. For the trip displayed in Fig. 1, one car-option and the 15 min train option are known a priori. The other train alternative is activated by the information service (see further below).

The participant's initial knowledge of the alternatives (i.e., before acquiring any information) is presented in the boxes below the black arrows. The following a priori knowledge is provided to the traveler for the known alternatives: for both car and train options, (1) best guesses for travel times and travel costs ${ }^{2}$ are provided, as well as (2) 'confidence' intervals, i.e. ranges of times and costs within which the participants are told (correctly) that actual values will fall almost certainly. A priori, train travelers do not know the exact departure times (although they do know the service's frequency), neither do they know whether or not a seat is available for them. Let us focus first on the situation where a traveler decides not to acquire information, but to directly embark on a trip by executing one of the known travel alternatives: a participant may start his journey by clicking one of the arrows, and subsequently confirming his choice in the appearing pop-up window. By confirming his or her choice, there is no possibility of adaptation until the interchange point is reached. Directly after confirmation, the traveler is confronted with the actual costs of the alternative, and train travelers were also informed whether or not they had a seat. As the trip commences (Fig. 1 shows the situation where a car option is chosen), the clock starts ticking and the arrow that represents the chosen alternative incrementally turns red, indicating the amount of distance traveled so far. These actual travel costs were drawn from a normal distribution, having the best guess as mean, and a quarter of the length of the 'confidence'

\footnotetext{
2 Travel times were framed as door to door for car-trips, and door-to-door minus waiting times for traintrips. All 'best guess' travel times were randomly varied beforehand and could take the values of 50 and $60 \mathrm{~min}$ for the car-option and 45 and $55 \mathrm{~min}$ for the train option (since to the latter also waiting times had to be added to get the total travel time). Travel costs were framed as fuel expenditures + parking costs for cartrips, and ticket tariffs for train-trips. Travel costs were randomly assigned beforehand the values of 3.5 euro or either 7 euro.
} 
interval as standard deviation, so that $95 \%$ of the drawn actual values would indeed fall within the 'confidence' interval. The same applies to the generation of actual travel times. Seat availability was randomly drawn from a discrete distribution $(50 \%$ chance of having a seat), waiting times from a uniform distribution between 0 and the headway (either 5 or $15 \mathrm{~min}$ ).

\section{The information service}

Let us focus now on the information acquisition options: as can be seen in Fig. 1, the information service's layout is an exact copy of the transport network. In the sample used for our current analyses all provided information was fully reliable, meaning that every received message corresponds to the actual value of that particular attribute. Participants were told about this complete reliability. The service presents three ways of acquiring information: firstly, a traveler may acquire information concerning one or more particular attributes of a known alternative, be it travel times and/or costs for a car or train option, and/or waiting times and seat availability for train options. This is done by first clicking on the arrow of a 'known' alternative and subsequently checking the boxes for those attributes for which information is needed (the information price is listed at every box, and varies between $0.15,0.30,0.45$ eurocents). After the boxes are checked, the service displays the information in the information box. Figure 1 presents the situation where for one of the car-options, both travel time and costs are informed about by the information service. Secondly, a traveler can ask the service to generate or activate one or more alternatives that are currently 'unknown' to him or her. This is done by clicking on an arrow within the information service screen that corresponds to an unknown alternative in the transport network. After this is done, a pop-up window appears that states the price of the information acquisition (being varied between 0.45, 0.60 and 0.75 eurocents) and asks for a confirmation. After confirmation, the alternative is made active, that is, its color turns black in the transport network, and the alternative can be executed from now on. Furthermore, the information service provides estimates for all the alternative's characteristics. These estimates are displayed in the box below the activated alternative in the information service screen part. Figure 1 presents such an information acquisition for the 5 min train option. Thirdly, a traveler may activate the socalled early-warning function. This function notifies a traveler when an alternative that is about to be chosen has an actual travel time that is substantially larger than the traveler's best guess (no strict level of travel time differences that triggers these messages is mentioned to the travelers). This type of information is acquired by clicking on a known alternative and checking the early warning box. Note that all these types of information can be acquired either pre-trip, as well during the trip or at the interchange point, which makes the information service 'mobile'. However, we focus on pre-trip information acquisition here. Note furthermore that the choice set size of information options depends on the choice set size of travel options: when a travel alternative is unknown, it can not be assessed in terms of its attributes; when it is known, it cannot be generated by the information service. Since there are more assessment options per known alternative than there are generation options per unknown alternative, the size of the information choice set is positively correlated with the size of the travel choice set. The early warning function is always available, irrespective the number of available travel alternatives in the traveler's travel choice set. 


\section{The visual aid}

In the lower right, pictures are placed that help the traveler identify with the situation at hand: several pictures were available to describe a number of possible pre- and in-trip situations for car (e.g. with and without congestion) as well as train (e.g. waiting at the station, being underway).

\section{Observed choices}

In total 4,536 recorded trips were made in the simulator in the presence of travel information. Of these trips, 2,454 were executed by car (54\%); the rest of times, train was chosen as a travel mode. In total, 5,298 unknown alternatives were generated (on average 1.17 times per trip). Travel time information was acquired 2,653 times (0.58), travel costs 1,023 times (0.23). Train departure time information was requested about 849 times (0.19), seat availability 303 times (0.07). The early warning function was activated for 1,252 trips (0.28).

Stated mode-choice experiment (observation of choices under complete knowledge)

This experiment consisted of a series of binary car-train mode-choices. Also here, trip purposes (the same ones as used in the simulator) were randomly assigned to trips. Car-alternatives were specified in terms of their travel times and costs, train alternatives additionally in terms of their waiting times and seat availability. Attribute values were varied systematically across alternatives. In order to avoid fatigue effects, a fractional factorial experimental design was applied that resulted in 16 binary choice tasks per individual. Attribute values were presented to participants as being certain. In total, $261 \times 16=4,176$ stated mode-choices were observed, of which 2,366 were choices for car $(57 \%)$, the remainder being train-choices. These observed choices were used to derive travelers' alternative-specific preferences as well as their valuation of travel and waiting times, costs and seat availability in trains.

\section{A measure of travel choice quality}

This section derives a measure of travel choice quality that, although it may be applied in a number of other settings, is geared for compatibility with our general notion of travel choice quality, the formulated hypotheses and the obtained dataset. Subsequently, the measure is applied to compute choice quality for the observed travel choices made under conditions of incomplete knowledge in the travel simulator environment.

\section{Derivation of a measure of travel choice quality}

Assume a traveler $i$ facing a set of available alternatives $S^{\text {avail }}$, containing $J$ alternatives $Y_{j}$ (say, mode-route combinations). Each of these alternatives consists of a bundle of relevant observed attributes. Assume for simplicity of presentation and without loss of generality that these attributes are invariable across travelers: $Y_{j}=\left\{x_{j 1}, x_{j 2}, \ldots\right\}$. Assume furthermore that the traveler has incomplete knowledge as (s)he is only aware of a 
subset of alternatives $\left(S_{i}^{\text {known }} \subset S^{\text {avail }}\right.$ ), and does not know the exact true values $\left\{\bar{x}_{j 1}, \bar{x}_{j 2}, \ldots\right\}$ of the bundle of relevant attributes $\left\{x_{j 1}, x_{j 2}, \ldots\right\}$. Rather, she perceives the bundle as a joint probability distribution $f_{i}\left(x_{j 1}, x_{j 2}, \ldots\right)$. Note that many other conceptualizations of the traveler's uncertain perception of these attributes would fit the argument being made here. We observe that a traveler, faced with this incomplete knowledge, chooses an alternative, say $Y_{i}^{\text {chosen }}=Y_{m}$, from $S_{i}^{\text {known }}$. To infer what alternative would have been chosen under hypothetical conditions of complete knowledge, take the same traveler, but assume now that she does know all available alternatives (i.e. $S_{i}^{\text {known }}=S^{\text {avail }}$ ), as well as the exact values of their relevant attributes. We assume that her choice under complete knowledge will be based on a compensatory utility structure, using her valuations $\beta_{i j 0}, \beta_{i j 1}, \beta_{i j 2}, \ldots$ of the true values $\left\{\bar{x}_{j 1}, \bar{x}_{j 2}, \ldots\right\}$ of the attributes of available alternatives:

$$
V_{i}\left(Y_{j} \in S^{\text {avail }}\right)=\beta_{i j 0}+\beta_{i j 1} \bar{x}_{j 1}+\beta_{i j 2} \bar{x}_{j 2}+\ldots
$$

Although it will often be practically infeasible to infer the exact values $\beta_{i j 0}, \beta_{i j 1}, \beta_{i j 2}, \ldots$ of travelers' preferences for alternatives and their attributes in the hypothetical situation of choice under complete knowledge, we may estimate proxies $\widehat{\beta}_{i j 0}, \widehat{\beta}_{i j 1}, \widehat{\beta}_{i j 2} \ldots$ for these preferences from other observed travel choices, made by the same travelers. ${ }^{3}$ Let us assume that this estimation process is performed within a random-utility framework, and that the variances of the i.i.d.-extreme value random utility components $\varepsilon_{i j}$ equal $\pi^{2} / 6$, i.e. that the scale of the utility is normalized to 1 (Ben-Akiva and Lerman, 1985). Under the requirement that (1) the choices used for preference-elicitation are observed in a choice-context that closely resembles the context within which the quality of travel-choices is evaluated, and (2) that both the observations made for parameter estimation, and the evaluation of travel-choice quality are made at very nearby moments in time, we assume that the scale of estimated proxies $\beta_{i j 0}, \widehat{\beta}_{i j 1}, \widehat{\beta}_{i j 2} \ldots$ equals the scale of true preferences $\beta_{i j 0}, \beta_{i j 1}, \beta_{i j 2}, \ldots$ Then, the deterministic utility of equation (1) can be rewritten into its random utility counterpart (2), where $\operatorname{var}\left(\varepsilon_{i j}\right)=\pi^{2} / 6$ :

$$
U_{i}\left(Y_{j} \in S^{\text {avail }}\right)=\widehat{\beta}_{i j 0}+\widehat{\beta}_{i j 1} \bar{x}_{j 1}+\widehat{\beta}_{i j 2} \bar{x}_{j 2}+\ldots+\varepsilon_{i j}
$$

This random-utility specification thus reflects the observational deficiencies of the analyst in terms of observing and predicting utilities of travel alternatives. Given this representation of a traveler's preferences (up to a random error), the analyst can predict choice probabilities for alternatives under conditions of complete knowledge by specifying the functional relationship between utility and choice, in this case resulting in a straightforward MNL-model. These choice probabilities represent the probability in the eyes of the analyst that the traveler, would she have complete knowledge, chooses an alternative, and is denoted $P\left(Y_{j} \mid S^{\text {avail }} ;\left\{\bar{x}_{k 1}, \bar{x}_{k 2}, \ldots\right\} \forall k \in S^{\text {avail }}\right)$. The assessment of travel-choice quality, in terms of whether the observed chosen alternative under incomplete knowledge corresponds to the

\footnotetext{
${ }^{3}$ Note that the availability of individual-level parameters is not critical for the argument made here, nor for the development of the measure of travel-choice quality that is proposed below. Obviously, parameters estimated at the individual level $\beta_{i j 0}, \beta_{i j 1}, \beta_{i j 2}, \ldots$ may provide a more reliable inference of an individual traveler's choice under hypothetical conditions of complete knowledge than group-level parameters $\beta_{j 0}, \beta_{j 1}, \beta_{j 2}, \ldots$, as the latter do not account for preference heterogeneity in the sample. However, substantial parts of this heterogeneity may be accounted for indirectly by having a number of socio-economic characteristics enter the utility functions.
} 
predicted choice probabilities under complete knowledge, now takes the form of the following likelihood function:

$$
L_{i}=\prod_{S_{i}^{\text {known }}}\left[P\left(Y_{j} \mid S^{\text {avail }} ;\left\{\bar{x}_{k 1}, \bar{x}_{k 2}, \ldots\right\} \forall k \in S^{\text {avail }}\right)^{y_{j} \mid S_{i}^{\text {known }}: f_{i}\left(x_{k 1}, x_{k 2}, \ldots\right) \forall k \in S_{i}^{\text {known }}}\right]
$$

Where $y_{j} \mid S_{i}^{\text {known }} ; f_{i}\left(x_{k 1}, x_{k 2}, \ldots\right) \forall k \in S_{i}^{\text {known }}=1$ if alternative $j$ is chosen under conditions of incomplete knowledge, and 0 otherwise. $L_{i}$ takes on values anywhere between 0 (poorest quality - the probability that the traveler would have chosen his current alternative had (s)he had complete knowledge is zero) and 1 (highest quality - the probability that the traveler would have chosen another alternative had (s)he had complete knowledge is zero). This range implies that our measure of travel choice quality is standardized with respect to a base-case and an upper bound.

It is important to note here, that the measure presented in equation (3) not only captures travelers' capability - under conditions of incomplete knowledge - to choose an alternative they would also choose had they had complete knowledge, but also the analyst's ability to approximate travelers' true preferences. This point can be clarified supposing that the analyst that has not been able at all to capture travelers' true preferences, i.e. signs and magnitudes of estimated parameters are completely off mark. As a result, the predicted choice probabilities are not representative of the choices of travelers under the hypothetical conditions of complete knowledge. Based on these erroneous predictions, low values of $L_{i}$ are likely to be found; these low values do not signal a low choice-quality from the side of the traveler, but rather inability from the side of the analyst. The same reasoning holds for the situation where the analyst does not find significant parameters in the first place, leading to insensitivity of predicted choice probabilities to changes in attribute values. The measure proposed in Eq. 3 therefore only has meaning within the context of the adopted theoretical framework, assuming that it provides a valid representation of actual choice processes. This is however not unique to the present measure: for example welfare-based accessibility measures also only have meaning within the context of the estimated model.

Furthermore, note that for analyses that do not focus on choice quality in an absolute sense, but rather in a relative one (such as analyses concerning the impact of information on choice quality), the measure presented in Eq. 3 does not require that the analyst knows all alternatives $S^{\text {avail. }}$ a minimum requirement for the application of the measure is that the analyst knows one more available alternative, besides the one chosen by the traveler. A fictive choice set $S^{\text {avail }}$ can then be constructed that contains the chosen alternative as well as the non-chosen but available alternative, and may be denoted $S^{\text {avail }} \subset S^{\text {avail }}$. Equation 3 then gives a travelchoice quality measure based on the predicted probability that the chosen alternative would also be the chosen one in the constructed subset $S^{\text {avail }}$. Finally, note that it is not necessary to assume that the analyst knows the true values $\left\{\bar{x}_{j 1}, \bar{x}_{j 2}, \ldots\right\}$ of all relevant attributes in $S^{\text {avail }}$ or $S^{\text {avail }}$. Rather, the analyst should know the true values of those attributes that she wishes to enter the analyses. Of course, the inclusion of only a very limited number of attributes in the analysis might lead to a less reliable measure of travel-choice quality.

Applying the measure of travel choice quality on the obtained dataset

Recall that (to avoid fatigue effects) it was decided to have participants make a number of 16 binary mode-choices under conditions of complete knowledge. As estimation of meaningful individual-level parameters $\widehat{\beta}_{i j 0}, \widehat{\beta}_{i j 1}, \widehat{\beta}_{i j 2}, \ldots$ is not possible based on these 16 
Table 2 estimation results (travel choices under complete knowledge)

\begin{tabular}{|c|c|c|}
\hline Variable & Parameter & t-Statistic (robust) \\
\hline \multicolumn{3}{|l|}{ Attributes of travel alternatives } \\
\hline Car constant & 2.139 & 5.257 \\
\hline Travel time car (min) & -0.063 & -15.088 \\
\hline Travel time train (min) & -0.046 & -12.130 \\
\hline Travel costs (euros) & -0.347 & -13.063 \\
\hline Travel time $\mathrm{x}$ Business (to be added to travel time parameter) & -0.049 & -5.464 \\
\hline Seat availability (on train) & 0.862 & 9.667 \\
\hline \multicolumn{3}{|l|}{ Socioeconomic variables } \\
\hline Age dummy (on car) & -0.027 & -7.461 \\
\hline Age dummy (to be added to travel costs parameter) & 0.002 & 3.243 \\
\hline Number of days car use in real life (on car) & 0.156 & 6.998 \\
\hline Number of days transit use in real life (on car) & -0.062 & -2.666 \\
\hline Education (on car) & -0.126 & -2.978 \\
\hline Dummy for "car is most important mode for me", (on car) & 0.524 & 4.006 \\
\hline Car license (on car) & 0.417 & 2.433 \\
\hline \multicolumn{3}{|l|}{ Model statistics } \\
\hline 0-Log-Likelihood & -2894.58 & \\
\hline Log-likelihood at convergence & -1716.46 & \\
\hline Adjusted rho-square (13 parameters) & 0.403 & \\
\hline Number of cases & 4176 & \\
\hline
\end{tabular}

observations per individual, group level parameters $\widehat{\beta}_{j 0}, \widehat{\beta}_{j 1}, \widehat{\beta}_{j 2}, \ldots$ were estimated and a number of socioeconomic characteristics were entered in the utility-specification in order to capture a substantial share of preference-heterogeneity in the sample. Table 2 presents the estimation results, obtained by estimating a binary logit model using the BIOGEME statistical package. ${ }^{4}$

Note that the scale-parameter is normalized to 1 , i.e. $\operatorname{var}\left(\varepsilon_{i j}\right)$ is set to $\pi^{2} / 6$. We will only briefly touch upon these results, as this study is not concerned with mode-choices. All parameters are of the expected sign, and significant. The car-constant implies that the average traveler in our sample prefers traveling by car over traveling by transit, all else being equal. The implied value of travel time is 10.89 euro per hour car travel time, and 7.95 euro per hour transit travel time (which itself is a sum of waiting time and in-vehicle time). For business trips, these values are 19.37 and 16.43 respectively. Seat availability in transit is positively valued, as expected. Concerning socio-economic characteristics, it appears that older travelers are more inclined to choose transit than younger ones, and have

\footnotetext{
${ }^{4}$ Note that, although multiple choices were observed per individual, we did not directly apply this panel structure in estimation. Acknowledging the panel structure through random agent effects and subsequent incorporation of these agent effects in our subsequent Structural Equation Model of the determinants of travel choice quality would greatly increase that model's complexity. For the present study, we have, for reasons of simplicity of presentation and for reasons of space limitations, chosen to indirectly acknowledge the panel structure by using robust t-statistics. Also, we have incorporated a number of sociodemographics in our models in order to increase our grasp of variation due to to observed, rather than unobserved factors.
} 
higher values of time as they value travel costs less negative than younger ones. Higher education (coded from 1 to 5 for increasing levels in the Dutch education system) leads to more transit use.

In order to obtain optimal congruence between on the one hand the contexts of the choices observed in the simulator (incomplete knowledge) and on the other hand the binary choices observed in the stated mode-choice experiment (complete knowledge), as was advocated in "Derivation of a measure of travel choice quality", it was decided to construct per observed choice in the simulator an artificial choice set $S^{\text {avail }} \subset S^{\text {avail }}$. This set contains the chosen alternative from $S_{i}^{\text {known }}$, as well as a randomly drawn alternative route from a different mode. That way, i.e. by making sure that the constructed set as well as the stated mode-choice set contain both one car and one rail alternative, we can apply the parameters estimated in a binary mode-choice context in order to predict choice probabilities in a similar binary mode-choice context, as much as possible avoiding potential incompatibilities with the scale of the utilities and the correlation structure of error components (IIA assumption). Together, this enables us to calculate the quality of each of the 4536 travel choices made in the simulator, as defined in Eq. 3 (note that we applied scale-factor 1 for the prediction of choice-probabilities, in congruence with the scale factor used in the estimation process of $\left(\widehat{\beta}_{i j 0}, \widehat{\beta}_{i j 1}, \widehat{\beta}_{i j 2} \ldots\right)$, and proceed with our analyses concerning the determinants of choice quality. Note that from an absolute point of view, this artificial choice set composition presents a bias in the measurement of choice quality. However, as elaborated on above, since we are in the remainder of this paper interested in choice quality in a relative sense, this bias does not affect our ability to study the relations between information use and quality of travel choices in an unbiased way.

\section{Empirical analysis of the impact of travel information on travel choice quality}

As can be seen in "Hypotheses on travel choice quality and the role of travel information', we hypothesize that the effects of a range of factors on travel choice quality may consist of direct and indirect effects. Take for example the factor 'uncertainty': it is hypothesized that the direct effect of this factor on choice quality is negative (relation 2). However, as we also hypothesize that travelers with limited knowledge will be relatively inclined to acquire information (relation 4) and that information acquisition leads to better choices (relation 3), we also hypothesize a positive indirect effect to exist (relation $3+4$ ). A confirmatory modeling technique that is well suited to deal with such a combination of direct and indirect effects is Structural Equation Modeling (SEM). As this linear-inparameters multivariate statistical modeling technique is fairly well known in transportation academia, and excellent in-depth treatments of the technique exist (e.g. Golob 2003), we will not discuss it in any details here. Table 3 presents the actual variables that enter our model. The result of the model estimation, using the LisRel package, is presented in Fig. 2. For simplicity of presentation, the error terms for the independent variables as well as the correlations between variables are suppressed in this figure. Estimated effects are mentioned, followed by their t-values. Effects with t-values of less than 1.96 (a two-tailed significance level of 0.05 ) are given dotted lines. The model's $\chi^{2}$ totaled 4.61, for 3 degrees of freedom, signaling a reasonable model fit $(P=0.203)$. Furthermore, most hypothesized relations appear significant and as will be discussed below, are of the expected sign. Note however, that only $4 \%$ of the variation in travel choice quality has been explained by variation in the independent variables, which is a rather low percentage. There are two 
Table 3 List of variables entering the structural equation model

\begin{tabular}{lll}
\hline $\begin{array}{l}\text { Knowledge at } \\
\text { the }\end{array}$ & $\begin{array}{l}\text { \#Known } \\
\text { alternatives }\end{array}$ & $\begin{array}{l}\text { The number of alternatives that is known at the outset of the trip, i.e. the } \\
\left.\text { number of alternatives in } S_{i}^{\text {known }} \text { (taking the values } 0,1,2,3,4\right)\end{array}$ \\
& $\begin{array}{l}\text { The level of uncertainty at the outset of the trip with respect to travel } \\
\text { times and costs (taking the values } 1 \text { (low), } 2 \text { (medium) and } 3 \text { (high } \\
\text { uncertainty)) }\end{array}$
\end{tabular}

\begin{tabular}{|c|c|c|}
\hline $\begin{array}{l}\text { Information } \\
\text { unreliability }\end{array}$ & $\begin{array}{l}\text { nformation } \\
\text { unreliability }\end{array}$ & $\begin{array}{l}\text { The standard deviation of travel time information (taking the values } 0 \\
\text { (fully reliable), } 5 \text { and } 10 \text { ) }\end{array}$ \\
\hline Trip purpose & Business trip & $\begin{array}{l}\text { Dummy variable: } 1 \text { IF the trip's purpose is a business meeting, } 0 \\
\text { otherwise }\end{array}$ \\
\hline Socioeconomic & Education $^{\mathrm{a}}$ & $\begin{array}{l}\text { Education level of participant, ranging from } 1 \text { (primary school only) to } 5 \\
\text { (university degree) }\end{array}$ \\
\hline Experimental & Trip Number ${ }^{b}$ & Dummy variable: 1 IF trip umber is between 8 and 15,0 otherwise. \\
\hline \multirow[t]{3}{*}{$\begin{array}{l}\text { Information } \\
\text { Acquisition }\end{array}$} & Early warning & $\begin{array}{l}\text { Dummy variable: } 1 \text { IF the early warning function was activated for this } \\
\text { trip, } 0 \text { otherwise }\end{array}$ \\
\hline & Assessment & $\begin{array}{l}\text { The total number of attributes that information is acquired about for this } \\
\text { trip }\end{array}$ \\
\hline & Generation & $\begin{array}{l}\text { The number of alternatives generated by the information service for this } \\
\text { trip }\end{array}$ \\
\hline $\begin{array}{l}\text { Travel choice } \\
\text { quality }\end{array}$ & $\begin{array}{l}\text { Quality } \\
\text { (Eq. 3) }\end{array}$ & $L=\prod_{S_{i}^{\text {known }}}\left[P\left(Y_{j} \mid S^{\text {avail }} ;\left\{\bar{x}_{k 1}, \bar{x}_{k 2}, \ldots\right\} \forall k \in S^{\text {avail }}\right)^{y_{j} \mid S_{i}^{\text {known }} f_{i}\left(x_{k 1}, x_{k 2}, \ldots\right) \forall k \in S_{i}^{\text {known }}}\right]$ \\
\hline
\end{tabular}

${ }^{a}$ It might be expected that education level influences the quality of observed travel choices

b Based on preliminary data-analyses, it was found that there exists a relation between the trip number and the choice quality, that appeared to persist, also when a number of other potential factors were controlled for. More specifically, it was found that the first couple of trips (up to about trip number 8), as well the last couple of trips (up from about trip number 16) had a lower average quality than the trips made in between. This may indicate that participants needed some time in order to understand the experiment (although 4 trips were made before the first trip was recorded for the analyses presented here), and suffered from fatigue effects after some number of trips. It was therefore decided to include trip number as an independent variable, in order to control for this potentially significant effect

c Note that information costs did not enter the SEM. This variable has been removed from the model after it became clear that its correlation with information acquisition (as operationalized in this study) was mostly insignificant. In other studies (e.g. Chorus et al. 2006e) we distinguished between each of the 40 information acquisition options and treated these separately in estimation, this correlation did appear significant. However, for the present study, such a disagregation appeared unfeasible due to the large number of additional variables involved

reasons why this limited explanatory power of the model is not surprising: firstly, as elaborated on in "Derivation of a measure of travel choice quality", it should be acknowledged that a substantial part of the variation in the applied likelihood measure might stem from the fact that proxies for the travelers' true preferences were used in predicting choice probabilities under the hypothetical conditions of complete knowledge, as true preferences could by definition not be derived under these hypothetical circumstances. Therefore, the analyst is by definition unable to determine exactly what alternative would have been chosen under conditions of complete knowledge, due to variation in the random error component in the utility function. This imperfect ability of the analyst results in an inherent measurement error with respect to the measurement of travel choice quality. This measurement results in additional variation in measured travel choice quality that cannot be explained by the reported hypothesized relations. Secondly, we only considered a limited number of explanatory variables, as our study is confirmatory in nature (how 


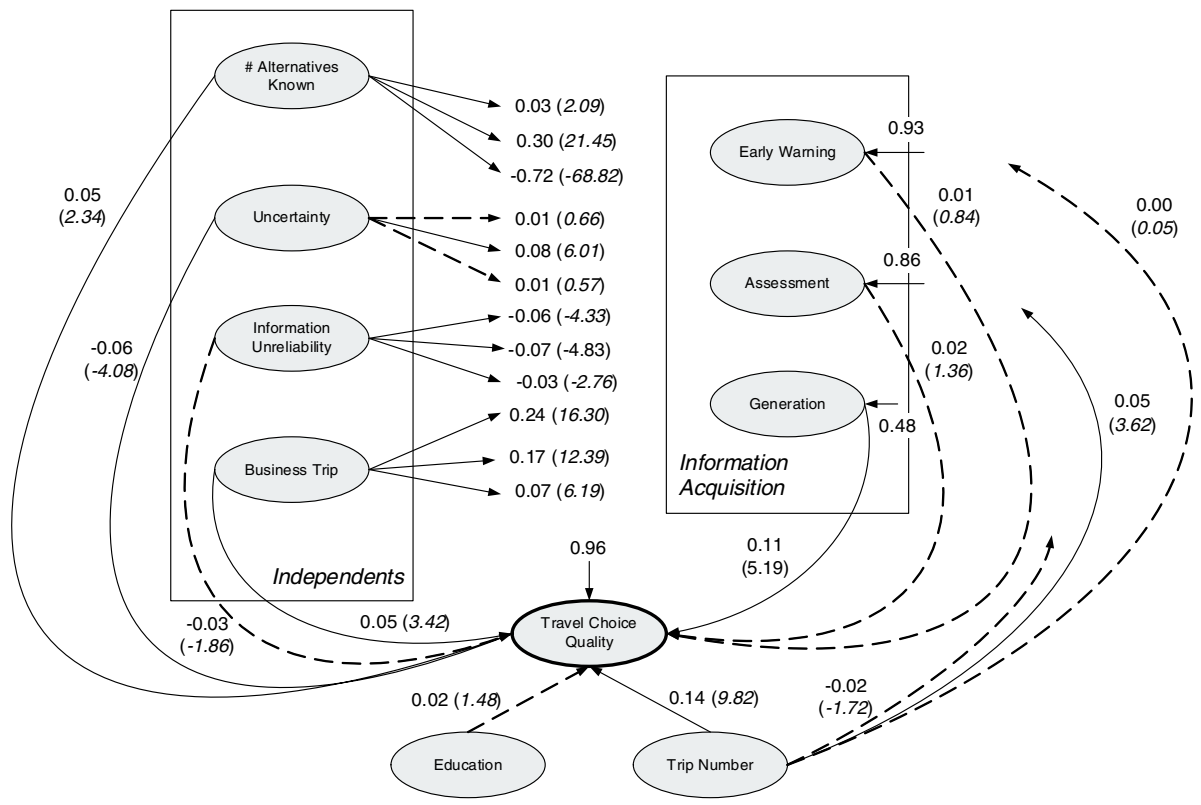

Fig. 2 A SEM of the impact of information on travel-choice quality

valid is the proposed measure of choice quality? And: does information acquisition lead to better travel choices?), rather than explanatory (what are the determinants of travel choice quality?): we are interested in investigating whether hypothesized relations appear significant in our model, rather than trying to identify all factors that may codetermine choice quality.

Turning to the hypotheses, formulated in "Hypotheses on travel choice quality and the role of travel information', the results of the SEM analysis indicate the following. First, the estimation results support hypotheses 1 and 2: the choice quality increases with the number of known alternatives and decreases with the uncertainty attached to the attributes of these alternatives ( $\mathrm{t}$-value 2.34 and -4.08 respectively). These effects were theoretically expected given the adopted general notion of choice quality and the fact that they appear to hold empirically provides evidence for the validity of the adopted general notion of choice quality and the measure of choice quality derived from it. We will proceed with testing the hypotheses that describe our intuition concerning the role of travel information in decision making processes and its effect on choice quality. Starting with relation 3, which is central to the study presented in this paper: the more information is acquired, the higher will be travel-choice quality. It appears that for all three information types, the sign of the effect of information acquisition on travel choice is positive. However, concerning the early warning function, the effect is insignificant at any reasonable level (t-value equals 0.84 ). A closer look at the working of this function explains the absence of a significant effect: the early warning function provides a warning in case travelers are about to embark on a trip on a route that has a substantially higher than expected travel time. Although this type of information may be helpful to travelers as it reduces the costs of thinking (Shugan 1980) and fits well within so-called satisficing decision-making strategies, the information content of the service is actually quite low. It says nothing about other than travel time-related 
attributes, and provides no actual messages of predicted travel time, just a warning. In this light, the insignificant effect on choice quality is not so surprising. With respect to the assessment of known alternatives, the effect is only significant at a one-tailed level of 0.10 (t-value equals 1.36), and not quite substantial. Apparently, by investigating the attributes of known alternatives alone, a traveler still remains quite prone to choosing an alternative that may not be optimal given the full set of available alternatives. However, the assessment type of information acquisition may help the traveler in increasing her travel-choice quality somewhat. The effect of generating previously unknown alternatives through the information services does have a highly significant and relatively large effect on choicequality (t-value equals 5.19). This signals the high information content of this information type: not only is an alternative added to one's choice set, estimates are provided for its attributes, too. That way, it increases the set of known alternatives and reduces the average level of uncertainty in this set. It is this double impact that apparently leads to an increase in travel-choice quality.

Concerning the hypothesized negative relation ( $\mathrm{nr} 4$ ) between knowledge and information acquisition, the following is found: the more alternatives a traveler knows of, the less inclined she will be to have an information service generate additional alternatives, as would be expected (t-value equals -68.82). It can also be seen that an increase in the number of known alternatives leads to an increase in information acquisition concerning the attributes of these alternatives (t-value 21.45) and an increase in the activation of the early warning function (t-value 2.09). This effect makes sense too, as explained in "Hypotheses on travel choice quality and the role of travel information": knowing more alternatives, with uncertainty attached to their attributes, means that there is more uncertainty to be reduced within the set of known alternatives. Finally, increasing levels of uncertainty per known alternative do lead, as hypothesized and found in literature, to more information acquisition concerning these attributes (t-value 6.01).

Moving to hypothesized relation 5, concerning business trips, it is found that, as was expected and often suggested in the literature, travelers are more inclined to acquire information on these arrival time sensitive trips (t-values 13.30, 12.39 and 6.19 for early warning, assessment and alternative generation respectively). It appears that especially the early warning function is an attractive option there, as it prevents arriving late, without forcing the traveler to embark on a time-consuming decision-making process for fast and reliable mode-route combinations. Moreover, it appears that when controlling for this increase in information acquisition, the quality of travel choices made for business trips is still higher than that of trips made for other purposes (t-value 3.42). This signals that individuals will put more effort in making a 'good choice' when the stakes are high. Indeed, we find that by giving the extra effort, they succeed in making better choices.

Relation 6 states that information unreliability has a double negative effect on choice quality, which appears to hold in our sample: firstly, it induces lower levels of information search (t-values equal $-4.33,-4.83,-2.76$ respectively for activating the early warning function, assessing known alternatives and generating unknown ones). Secondly, information that is acquired has, to the extent that it is unreliable, a lower potential to reduce uncertainty and increase choice-quality (t-value equals -1.86 ). These results signal the paramount importance of the reliability of information services. A slight positive effect of education level on choice quality is found, though the effect is not significant at high levels (t-value 1.48). Finally, it appears that as was expected, participants made relatively poor choices in the beginning and the end of the experiment (t-value for trips made halfway the 
experiment equals 9.82). Although we controlled for this factor in model estimation, it would have been wise to limit the duration of the experiment to less than the two and a half hours we took.

\section{Conclusions and discussion}

This paper investigated the impact of travel information on the quality of travel choices. It distinguishes itself from earlier studies on this topic by empirically investigating the impact of a variety of travel information types, including the assessment of known alternatives and the generation of unknown alternatives, on the quality of observed multimodal travel choices, using objective rather than subjective measurements. Furthermore, the potential impact of travel information is considered along multiple attribute-dimensions of alternatives, such as travel times and travel costs of car and transit alternatives, as well as waiting times and seat probabilities in transit. Data needed for empirical analysis is obtained from a choice experiment under conditions of incomplete knowledge and information provision in a multimodal travel simulator, in combination with a web-based modechoice experiment under conditions of complete knowledge.

A Structural Equation Model is estimated that is based on computed values of travel choice quality for each of the 4,536 trips that were made under conditions of incomplete knowledge. Estimation results show that theoretical expectations with respect to the workings of the adopted general notion of travel choice quality appear to hold empirically, providing support for the validity to this general notion and the proposed measure derived from it. Simultaneously with this validation, a number of other hypothesized relations was tested, referring to the role of travel information acquisition in travelers' decision-making processes and its influence on travel choice quality. It is found that the more alternatives a traveler knows, the less she will be inclined to have an information service generate additional alternatives, as would be expected. However, an increase in the number of known alternatives leads to an increase in information acquisition concerning the attributes of these alternatives and an increase in the activation of the early warning function. Increasing levels of uncertainty per known alternative lead, as hypothesized, to more information acquisition. Concerning the effect of acquired information on choice-quality, it is found that the early warning function has a too low level of information content to substantially increase choice-quality. By investigating the attributes of known alternatives alone, a traveler also remains quite prone to choosing an alternative that may not be optimal given the full set of available alternatives. The effect of generating previously unknown alternatives through the information services does have a highly significant and relatively large effect on choice-quality. Furthermore, it appears that on business trips, also when controlling for the increase in information acquisition for these trips, travel choicequality is higher than on trips made for other purposes, signaling that individuals put more effort in making a 'good choice' when the stakes are high. Information unreliability appears to have a double negative effect on choice quality: it induces lower levels of information search, and information that is acquired has a lower potential to reduce uncertainty and increase choice-quality. The fact that all found relations in the SEManalysis are either intuitive or well interpretable indicates that the potential issue of confounding between choice quality and the analyst's capability to derive reliable preferences of travelers (see "Derivation of a measure of travel choice quality') did not play a too substantial role given the present dataset. 
Given these results, this study provides an indication that claims of a positive impact of travel information on travel-choice quality are, at least to some extent, justified. The size of the impact, however, differs between types of information. A relatively large effect is generated by information services that provide travelers with previously unknown alternatives simultaneously with the provision of estimates of their attributes. Furthermore, as found effects are well explainable and mostly significant, the study provides evidence of the applicability of the proposed measure as a means to study the impact of travel information on travel choice quality.

However, it is important to note here again that the sample used for analysis is not a random one: we invited individuals having experience with both travel by transit and private car. The above stated conclusions should therefore be interpreted with caution, and must certainly not be generalized directly towards the full population of travelers. In particular, our findings should not be interpreted to suggest that providing captive cardrivers or transit users with information on other modes would enhance the quality of their choices. Further study efforts, preferably based on revealed travel choice data from actual travel situations involving random samples of travelers, are needed to replicate the findings presented here and test their validity.

Acknowledgments This paper has been written in the context of the PITA program, which is a collaboration between the Delft University of Technology and the Eindhoven University of Technology, and sponsored by NWO/Connekt. We would like to take the opportunity to thank three anonymous referees for providing valuable suggestions for improvement of an earlier version of paper.

\section{References}

Abdel-Aty, M., Abdalla, M.F.: Modeling drivers' diversion from normal routes under ATIS using generalized estimating equations and binomial probit link function. Transportation 31 (3), 327-348 (2004)

Adler, J.L., Blue, V.J.: Toward the design of intelligent travel information systems. Trans. Res. 6C,157-172 (1998)

Adler, J.L.: Investigating the learning effects of route guidance and traffic advisories on route choice behavior. Trans. Res. Part C 9(1), 1-14 (2001)

Al-Deek, H.M., Khattak, A.J., Thananjeyan, P.: A combined traveler behavior and system performance model with advanced traveler information systems. Trans. Res. Part A 32(7), 479-493 (1998)

Arentze, T.A., Timmermans, H.J.P.: Information gain, novelty seeking and travel: a model of dynamic activity-travel behaviour under conditions of uncertainty. Trans. Res. Part A 39(2-3), 125-145 (2005)

Arnott, R., de Palmam, A., Lindsey, R.: Does providing information to drivers reduce traffic congestion? Trans. Res. Part A 25(5), 309-318 (1991)

Avineri, E., Prashker, J.N.: Sensitivity to uncertainty: need for a paradigm shift. Trans. Res. Rec. 1854, 9098 (2003)

Bates, J., Polak, J., Jones, P., Cook, A.: The valuation of reliability for personal travel. Trans. Res. 37E, 191-229 (2001)

Ben-Akiva, M., Lerman, S.R.: Discrete choice analysis: theory and application to travel demand. The MIT Press, Cambridge (1985)

Ben-Akiva, M., De Palma, A., Kaysi, I.: Dynamic network models and driver information systems. Trans. Res. 25A, 251-266 (1991)

Benjamin, J.M.: A study of the impact of APTS on service quality perceptions of elderly and disabled riders. J. Public Trans. 9(1), 1-18 (2006)

Bogers, E.A.I., Viti, F., Hoogendoorn, S.P.: Joint modelling of ATIS, habit and learning impacts on route choice by laboratory simulator experiments. Forthcoming in Transportation Research Records (2005)

Bonsall, P.: Predicting travellers' response to uncertainty. In: Hensher (eds.) Travel Behaviour Research: the Leading Edge. Pergamon, Amsterdam (2001)

Bonsall.: Motivating the respondent: how far should you go? In: Mahmassani, H.S. (eds.) In perpetual motion: travel behavior research opportunities and application challenges. Oxford, UK (2002) 
Bonsall, P.: Traveller behaviour: decision-making in an unpredictable world. J. Intell. Trans. Syst. 8, 45-60 (2004)

Carson R.T., Groves T., Machina M.J.: Incentive and informational properties of preference questions. Working paper (2000)

Chatterjee, K., McDonald, M.: Effectiveness of using variable message signs to disseminate dynamic traffic information: evidence from fiels trials in European cities. Trans. Rev. 24, 559-585 (2004)

Chorus, C.G., Molin, E.J.E., van Wee, G.P.: Use and effects of Advanced Traveller Information Services (ATIS): a review of the literature. Trans. Rev. 26(2), 127-149 (2006a)

Chorus, C.G., Arentze, T.A., Molin, E.J.E., Timmermans, H.J.P.: The value of travel information: decisionstrategy specific conceptualizations and numerical examples. Trans. Res. Part B 40(6), 504-519 (2006b)

Chorus, C.G., Molin, E.J.E., Arentze, T.A., Hoogendoorn, S.P., Timmermans, H.J.P., Van Wee, G.P.: Observing the making of travel choices under uncertainty and information: validation of travel simulator. Paper presented at the 85th annual meeting of the Transportation Research Board, Washington, D.C. $(2006 \mathrm{c})$

Chorus, C.G., Molin, E.J.E., Arentze, T.A., Timmermans, H.J.P., van Wee, G.P.: Travelers' need for information in traffic and transit: results from a web survey. J. Intell. Trans. Syst. (forthcoming)

Chorus, C.G., Walker, J.L., Ben-Akiva, M.E.: Travel information use and effects. Paper presented at the 11th conference of the International Association of Travel Behavior Research, Kyoto (2006d)

Commission of the European Communities. European transport policy for 2020: Time to decide. Luxembourg, Luxembourg (2001)

Denant-Boèmont, L., Petiot, R.: Information value and sequential decision-making in a transport setting: an experimental study. Trans. Res. 37B, 365-386 (2003)

Department for Transport. The future of transport: a network for 2030. London, UK (2004)

Emmerink, R.H.M., Axhausen, K.W., Nijkamp, P., Rietveld, P.: Effects of information in road transport networks with recurrent congestion. Transportation 22, 21-53 (1995)

Emmerink, R.H.M., Nijkamp, P., Rietveld P., Van Ommeren, J.N.: Variable message signs and radio traffic information: an integrated empirical analysis of drivers' route choice behaviour. Trans. Res. 30A, 135153 (1996)

Fayish, A.C., Jovanis, P.P.: Usability study of statewide web-based roadway weather information systems. Paper presented at the 83rd meeting of the Transportation Research Board, Washington, D.C. (2004)

Gentile, G., Nguyen, S., Pallottino, S.: Route choice on transit networks with online information at stops. Trans. Sci. 39(3), 289-297 (2005)

Golledge, R.G.: Dynamics and ITS: behavioural response to information available from ATIS. In: Mahmassani H.S. (eds), In perpetual motion: travel behaviour research opportunities and application challenges. Pergamon, Amsterdam (2002)

Golob, T.F.: Structural equation modeling for travel behavior research. Trans. Res. Part B 37(1), 1-25 (2003)

Hato, E., Taniguchi, M., Sugie, Y., Kuwahara, M., Morita, H.: Incorporating an information acquisition process into a route choice model with multiple information sources. Trans. Res. 7C, 109-129 (1999)

Hoogendoorn-Lanser S., Van Nes R.: Multi-modal choice set composition: analysis of reported and generated choice sets. Paper presented at the 83rd meeting of the Transportation Research Board, Washington, D.C. (2004)

Horst, R.S.N. van der, Ettema, D.: Use of travel information and effects on mode choice for recreational trips. Paper presented at the 84th meeting of the Transportation Research Board, Washington, D.C. (2005)

Jou, R., Lam, S., Liu,Y., Chen, K.: Route switching behavior on freeways with the provision of different types of real-time traffic information. Trans. Res. Part A 39, 445-461 (2005)

Khattak, A.J., Schofer, J.L., Koppelman, F.S.: Commuters' enroute diversion and return decisions: analysis and implications for advanced traveller information systems. Trans. Res. 27A, 101-111 (1993)

Khattak, A.J., Yim, Y., Prokopy, L.S.: Willingness to pay for travel information. Trans. Res. 11C, 37-159 (2003)

Levinson, D.: The value of advanced traveler information systems for route choice. Trans. Res. 11C, 75-87 (2003)

Mahmassani, H.S., Jayakrishnan, R.: System performance and user response under real-time information in a congested traffic corridor. Trans. Res. Part A 25(5), 293-308 (1991)

Ministry of Transport, Public Works and Water Management. De markt voor multi-modaal personenvervoer. Rijkswaterstaat, Rotterdam, The Netherlands (in Dutch) (2002)

Molin E.J.E., Chorus C.G.: Willingness to pay for personalized public transport information services. Paper presented at the 83rd meeting of the Transportation Research Board, Washington, D.C. (2004) 
Ouwersloot, H., Nijkamp, P., Pepping, G.: Advanced elematics for travel decisions: a quantitative analysis of the stopwatch project in Southampton. Environ. Planning Part A 29(6), 1003-1016 (1991)

Payne, J.W., Bettman, J.R., Johnson, E.J.: The adaptive decision maker. Cambridge University Press, Cambridge (1993)

Peirce, S., Lappin, J.: Why don't more people use advanced traveller information? Evidence from the Seattle area. Paper presented at the 83rd meeting of the Transportation Research Board, Washington, D.C. (2004)

Petrella, M., Lappin, J.: Los Angeles and Seattle: a comparative analysis of customer response to online traffic information. Paper presented at the 83rd meeting of the Transportation Research Board, Washington, D.C. (2004)

Polak, J., Jones, P.: The acquisition of pre-trip information: a stated preference approach. Transportation 20 , 179-198 (1993)

Polydoropoulou, A., Gopinath, D.A., Ben-Akiva, M.: Willingness to pay for advanced traveller information systems: Smartraveller case study. Trans. Res. Rec. 1588, 1-9 (1997)

Ramming, M.S.: Network knowledge and route choice. PhD-thesis, Massachusetts Institute of Technology. Cambridge, MA (2002)

Rietveld, P., Bruinsma, F.R., van Vuren, D.J.: Coping with unreliability in public transport chains: a case study for Netherlands. Trans. Res. 35A, 539-559 (2001)

Shugan S.M.: The cost of thinking. J. Consum. Res. 7, 99-111 (1980)

Srinivisan, K., Chen, I., Reddy, P., Jovanis, P.P.: Pre-trip information systems: an investigation into users' information acquisition process. Paper presented at the 78th meeting of the Transportation research Board, Washington, D.C. (1999)

Szeto, W.Y., Lo, H.K.: The impact of advanced traveler information services on travel time and schedule delay costs. J. Intell. Trans. Syst. 9(1), 47-55 (2005)

Targa, F., Khattak, A.J., Yim, Y.: Understanding access and use of dynamic travel information. Paper presented at the 82nd meeting of the Transportation Research Board, Washington, D.C. (2003)

Walker, J.L., Ben-Akiva, M.E.: Consumer response to traveler information systems: laboratory simulation of information searches using multimedia technology. ITS J. 3, 1-20 (1996)

Yim, Y., Khattak, A.J.: Traveller response to new dynamic information sources: analyzing corridor and area-wide behavioural surveys. Paper presented at the 81 st meeting of the Transportation Research Board, Washington, D.C. (2002)

\section{Author biographies}

Caspar Chorus holds a PhD in Technical Sciences (cum laude) from Delft University of Technology, and is currently an Assistant Professor at Eindhoven University of Technology's Urban Planning Group. His general interests include traveler behavior research / decision making under knowledge limitations / discrete choice analysis.

Theo Arentze received a Ph.D. in Decision Support Systems for urban planning from the Eindhoven University of Technology. He is now an Associate Professor at the Urban Planning Group at the same university. His main fields of expertise and current research interests are decision support systems, activitybased modeling, discrete choice modeling, knowledge discovery and learning-based systems with applications in urban and transport planning.

Harry Timmermans received a Ph.D. in Spatial Sciences from the University of Nijmegen. He is Chair of the Urban Planning Group and Director of the European Institute of Retailing and Consumer Services. His main fields of expertise concern behavioral modeling, consumer studies and computer systems in a variety of application contexts including transportation. 